\title{
Erythroleukemia in Remission
}

National Cancer Institute

\section{Source}

National Cancer Institute. Erythroleukemia in Remission. NCI Thesaurus. Code C8560.

Erythroleukemia that is responding to therapy, i.e. It is not progressing and possibly regressing. 